\title{
Tracheoesophageal Voice Prosthesis Outcomes: Success or Insucess?
}

\author{
Sara Cruz, Rita Viana, Joana Guimarães, João Fernandes, Joaquim Castro Silva, Eurico Monteiro \\ Serviço de Otorrinolaringologia, Instituto Português de Oncologia Francisco Gentil do Porto, Porto, Portugal \\ Email: saramadalena@hotmail.com
}

Received September 30, 2013; revised October 30, 2013; accepted November 10, 2013

Copyright (C) 2014 Sara Cruz et al. This is an open access article distributed under the Creative Commons Attribution License, which permits unrestricted use, distribution, and reproduction in any medium, provided the original work is properly cited. In accordance of the Creative Commons Attribution License all Copyrights (C) 2014 are reserved for SCIRP and the owner of the intellectual property Sara Cruz et al. All Copyright (c) 2014 are guarded by law and by SCIRP as a guardian.

\section{ABSTRACT}

Introduction: Total laryngectomy/pharingo-laryngectomy is a potentially aggressive surgery for advanced laryngeal/hypopharyngeal carcinomas, which results in important physical and functional changes that compromise some of the most vital functions, including speech communication. For these patients, tracheoesophageal speech is considered to be the gold standard for voice rehabilitation. Objectives: The purpose of the present study was to determinate the success rate of voice prosthesis rehabilitation, voice prosthesis lifetime and the rate of complications, and its related clinicopathologic factors. Material and Methods: Retrospective review of 92 patients who undergone tracheoesophageal puncture (TEP) performed between January 2007 and December 2012 at the Francisco Gentil Portuguese Institute of Oncology of Oporto. Age, primary disease, staging, the extent of surgical resection, radiotherapy treatment, timing of TEP, surgical and prosthesis-related complications were noted. The impact of these clinicpathological factors on functional outcome, complications of TEP and lifetime of prosthetic valves was assessed in univariate analysis. Vocal rehabilitation efficacy with voice prosthesis was assessed with the multidimensional Harrison-Robillard Shultz (HRS) Rating Scale. Lifetime of voice prosthesis and early and late complications were recorded. Results: A total of 83 patients met the study criteria, predominantly males (94\%) with a mean age of 63.7 years. $77 \%$ of the patients underwent primary and $23 \%$ secondary TEP. $68.7 \%$ of patients achieved functional tracheoesophageal speech (HRS score $\geq 10$ ), 67.2\% had performed primary TEP and $73.7 \%$ had performed secondary TEP. The mean device lifetime was 9.8 months for voice prosthesis. Prosthesis-related complications occurred in $81 \%$ of the patients and the most common issues were prosthesis leakage $(76 \%)$ and displacement $(22 \%)$. The most common surgical-related problem was a large and deep tracheostoma. Conclusions: Our success rate of voice rehabilitation was comparable to that reported in published literature with a satisfactory median device lifetime. Because of its safety and simplicity, tracheoe- sophageal puncture is considered to be an effective method for voice rehabilitation after total laryngectomy.

\section{KEYWORDS}

Laryngeal Cancer; Total Laryngectomy; Tracheoesophageal Puncture; Tracheoesophageal Speech; Voice Prosthesis

\section{Introduction}

There has been significant effort in the development of organ-preservation therapies, such as partial reconstructive surgery, radiation therapy alone, induction chemotherapy or concurrent chemoradiotherapy with acceptable results in advanced laryngeal cancer. However, total laryngectomy (TL) or pharyngolaryngectomy (TPL) still remains the best approach in patients with advanced la- ryngeal/hypopharyngeal carcinoma [1].

It is potentially an aggressive surgery resulting in important physical and functional changes that compromise some of the most vital functions, including speech communication. These changes may have a profound adverse impact on the patient's physical, functional, and psychological health that may result in a decreased quality of life. 
Speech restoration is an important part of functional rehabilitation for patients undergoing total laryngectomy and can be achieved by the use of esophageal speech, an artificial larynx, or creation of a tracheoesophageal fistula to place a voice prosthesis, a method that has become popular because of its introduction by Singer and Blom in 1980 [2-4].

The advantages of prosthesis devices are numerous and include immediate voice production, high success rates (50\% to $90 \%$ ), and the possibility of sustained speech with a more fluent quality than that with esophageal speech. Consequently, in many centres, tracheoesophageal puncture (TEP) with voice prosthesis placement has replaced esophageal speech as the gold standard for voice rehabilitation $[5,6]$.

The wide variability of reported success rates of the TEP procedure may be explained by the lack of controlled success criteria. Almost every published study used its own success criteria, however in 1992, Harrison and Robillard-Shultz introduced the Harrison-RobillardShultz Tracheoesophageal Puncture Rating Scale, a 15point rating scale incorporating key elements of the definitions of use and quality of speech as well as ability to care for the fistula and the prosthesis from 15 reviewed studies [6].

TEP can be performed at the time of TL (primary TEP) or afterwards (secondary TEP) [1]. The advantage of primary TEP placement is that patients are not subjected to a second operation for the acquisition of voice and may initiate voice rehabilitation within 2 weeks of the laryngectomy. On the other hand, primary TEP is thought to be associated with an increased risk of surgical and stoma-related complications, such as fistula, leakage at the puncture site, stomal stenosis, and local infection. It has been suggested that the complication rate may be decreased if TEP is performed as a secondary procedure due to increased duration of tracheostomal healing. Moreover, secondary TEP patients may have more reasonable expectations regarding the quality of tracheoesophageal speech and therefore be more satisfied with their voice following the period of postoperative aphonia [4].

The aims of the present study were to determine the success rate of voice prosthesis rehabilitation, voice prosthesis lifetime and the rate of complications, and analyse related clinicopathologic factors by comparing the results of primary and secondary TEP.

\section{Material and Methods}

\subsection{Patients}

A retrospective review of 92 patients with laryngeal or hypopharyngeal carcinomas who underwent to tracheoesophageal puncture with insertion of voice prosthesis at the time (primary TEP) or after (secondary TEP) total laryngectomy/pharyngolaryngectomy, between January 2007 and December 2012, at the Francisco Gentil Portuguese Institute of Oncology of Oporto. The exclusion criteria were patients that died before 6 months after TEP, patients with a period of vocal rehabilitation less than 6 months or with incomplete information medical records. A total of 9 patients were excluded from the basis of this review.

The data collected resulted from the analysis of medical records relatively to clinical history, physical examination, imaging studies (computed tomography, magnetic resonance imaging), surgical findings, pathology reports, complementary treatments and speech therapy.

Were evaluated the following parameters: patient demographic factors and clinical outcomes (age, sex, tobacco use, symptoms, comorbidity, primary disease, staging), treatment (extent of surgical ressection, reconstruction, neck dissection, complementary treatment), vocal rehabilitation (timing of TEP, type of voice prosthesis used, first and subsequent prosthesis lifetime, number of valve replacements, surgical and prosthesisrelated complications and their management, HRS scale, functional outcome, accessory devices). The impact of these clinicpathological factors on functional outcome, complications and lifetime of prosthetic valves was assessed in univariate analysis.

Comorbidity of patients was determined by the ASAscore obtained from the preoperative records. Patients with pharyngeal or laryngeal carcinoma were staged according to the 2010 American Joint Committee on Cancer (AJCC) staging system.

\subsection{Tracheoesophageal Puncture and Voice Prosthesis}

Before TEP, patients had undergone psycological evaluation and had been given the opportunity to meet voice rehabilitated patients. Those with poor motivation or diseases (mainly neurological and/or pneumological) prone to putative prosthesis handling problems had not been considered for voice prosthesis placement.

In patients who underwent secondary TEP, the vibratory pattern of the pharyngoesophageal segment (PES) was assessed by an air esophageal insuflation test as described by Blom et al. Patients with hypertonicity or spasm of the PES did not undergo voice prosthesis insertion.

The voice prosthesis used were Provox ${ }^{\circledR}$, Provox $2 \AA$ or Provox Vega ${ }^{\circledR}$. In patients with primary TEP a cricopharyngeal myotomy was indicated at the time of TL.

Postoperative complications as pharyngocutaneous fistula, cervical cellulitis, cervical infection, respiratory infection were considered in the analysis. 
To the calculations of the lifetime of the prosthetic valves was used the period between the date of insertion and the date of removal of the voice prosthesis.

\subsection{Surgical and Prosthesis-Related Complications}

Complications were divided in surgical and prosthesisrelated complications.

Surgical-related complications of interest were the development of a large and deep tracheotomy hole, cutaneous infection and pharyngoesophageal stricture.

Prosthesis-related complications were due to voice prosthesis problems or TEP problems. These included leakage either through or around the prosthesis, granulation tissue, dislodgment of prosthesis and aspiration.

When complications occurred, interventions included changes in size or type of prosthesis, insertion of nasogastric tube, granulation tissue cauterizing, antifungal rinses administrations and bronchoscopic removal of aspirated prosthesis. Spontaneous closure of tracheoesophageal fistula after dislodgment of prosthesis and surgical closure of TEP due to intractable leakage were recorded.

\subsection{Vocal Rehabilitation}

Each patient received voice prosthesis rehabilitation training by a Speech Therapist approximately 2 weeks after voice prosthesis placement that observed the patients for about 6 months or longer.

Functional outcomes were recorded using the multidimensional Harrison-Robillard Shultz (HRS) Rating Scale, for about 6 months postoperatively or longer. The HRS scale defines success by three parameters scored on a 1 - 5 points scal: use (degree to which tracheoesophageal speech is used as the main means of communication), quality (the ease of voice production and its effect on intelligibility), and care (degree of patient independence from professional aid for maintenance of the fistula and the prosthesis). An overall score of 12 or higher is considered successful voice prosthesis rehabilitation.

In our study, the maximum HRS score was 13 instead of 15 because the indwelling voice prosthesis is not to be self-removed or inserted by patient and the hands-free kit, is not accessible to most users due to economic reasons, even if patients have indication to use it, so the maximum reachable score of the subscale parameter quality care and use in the majority of these patients was only 4 instead of 5 points. An overall score of 10 or higher was considered to represent successful voice prosthesis rehabilitation.

The impact of the voice impairment in the patient's life was assessed with the Voice Handicap Index (VHI).
The quality of the TE voice was evaluated perceptually by a speech therapist on the basis of a conversational speech sample. TEP voices were classified as excellent, fair, or poor, based primarily on vocal duration, continuity, fluency, and vocal loudness during conversational speech.

Patients without functional speech and documented hypertonicity of PES by air esophageal insufflation test were submitted to a diagnostic blockade of the pharyngeal plexus with $1 \%$ lidocain, to establish whether a short-term pharmacological denervation of the constrictor pharyngeal muscles would solve the problem.

\subsection{Data Analysis}

Statistical analyses were performed using Qui2 test, confirmed by Fisher's exact test and non-parametric MannWhitney's test for qualitative variables and T-Student test and Wilcoxon test to quantitative variables. Prosthetic valve survival curves were estimated with the Kaplan-Meier method and compared with the log-Rank test. A Cox proportional hazards model, using a forward variable selection technique, was used to identify the independently predictive variables of prosthetic valve failure. All statistical tests were performed with the SPSS Statistical Software, version 17.0 and p values of less than 0.05 were regarded as statistically significant.

\section{Results}

\subsection{Patient Demographics}

A total of 83 patients were considered for this evaluation (Table 1). There were 78 men (94\%) and 5 women (6\%) ranging in age from 39 to 85 years (mean, 63.7 years).

Laryngeal carcinoma was present in 72 patients (86.7\%), and hypopharyngeal carcinoma in 11 patients (13.3\%). According to the AJCC staging system, there were 0 patients $(0 \%)$ with stage I disease, 1 patient $(1.2 \%)$ with stage II disease, 29 patients (34.9\%) with stage III disease, and 53 patients (63.9\%) with stage IV disease.

\subsection{Treatment}

Sixty-three patients (75.9\%) underwent TL, 20 patients (24.1\%) underwent TPL with primary closure of the pharyngeal defect. All patients had simultaneous neck dissection (Table 1).

Sixteen patients (19.3\%) had surgery only as the definitive treatment. Adjuvant postoperative therapy was used 67 patients (80.7\%). Radiotherapy (RT) alone or combined with chemotherapy (QT) was performed in 66 patients (79.5\%), postoperatively in sixty-three (75.9\%) patients. Twenty-five patients (30.1\%) efectuate QT/RT (Table 1). 
Table 1. Clinicopathologic factors and functional outcome of tracheoesophageal puncture speech rehabilitation.

\begin{tabular}{|c|c|c|c|c|}
\hline & Total & Primary TEP & Secondary TEP & $P$ value \\
\hline Patients no. (\%) & $83(100)$ & $64(77.1)$ & 19 (22.9) & \\
\hline Age (years) & & & & 0.27 \\
\hline$<60$ & $26(31.3)$ & $22(34.4)$ & $4(21.1)$ & \\
\hline$\geq 60$ & $57(68.7)$ & $42(65.6)$ & 15 (78.9) & \\
\hline Sex no. (\%) & & & & 0.35 \\
\hline Women & $78(94)$ & $3(4.7)$ & $2(10.5)$ & \\
\hline Male & $5(6)$ & $61(95.3)$ & 17 (89.5) & \\
\hline Comorbidity no. (\%) & & & & 0.31 \\
\hline $\mathrm{ASA} \leq 3$ & $64(77.1)$ & $51(79.7)$ & $13(68.4)$ & \\
\hline ASA $>3$ & $19(22.9)$ & $13(20.3)$ & $6(31.6)$ & \\
\hline Tumor site no. (\%) & & & & 0.14 \\
\hline Larynx & $72(86.7)$ & $58(90.6)$ & 14 (73.7) & \\
\hline Hypopharynx & $11(13.3)$ & $6(9.4)$ & $4(21.1)$ & \\
\hline Staging no. (\%) & & & & 0.25 \\
\hline$\leq \mathrm{III}$ & $30(36.1)$ & $21(32.8)$ & $9(47.4)$ & \\
\hline$>$ III & $53(63.9)$ & $43(67.2)$ & $10(52.6)$ & \\
\hline Resection no. (\%) & & & & 0.8 \\
\hline $\mathrm{TL}$ & 63 (75.9) & $49(76.6)$ & $14(73.7)$ & \\
\hline PLT & $20(24.1)$ & $15(23.4)$ & $5(26.3)$ & \\
\hline Neck dissection no. (\%) & & & & 0.49 \\
\hline Functional & $70(84.3)$ & $53(82.8)$ & 17 (89.5) & \\
\hline Radical & $13(15.7)$ & $11(17.1)$ & $2(10.5)$ & \\
\hline Complementary treatment no. (\%) & & & & 0.88 \\
\hline None & $16(19.3)$ & $10(15.6)$ & $6(31.6)$ & \\
\hline $\mathrm{RT}$ & $41(49.4)$ & 33 (51.6) & $8(42.1)$ & \\
\hline QT & $1(1.2)$ & $1(1.6)$ & $0(0.0)$ & \\
\hline $\mathrm{QT} / \mathrm{RT}$ & $25(30.1)$ & $20(31.3)$ & $5(26.3)$ & \\
\hline Radio therapy no. (\%) & & & & 0.55 \\
\hline Preoperative & $3(3.6)$ & $2(3.1)$ & $1(5.3)$ & \\
\hline Postoperative & $63(75.9)$ & $51(79.7)$ & $12(63.2)$ & \\
\hline \multicolumn{5}{|l|}{ Vocal Lifetime (meses) } \\
\hline Total voiceprothesis & 9.8 & 10.4 & 8.0 & 0.01 \\
\hline First voiceprothesis & 11.6 & 12.3 & 9.4 & 0.01 \\
\hline Subsquent voiceprothesis & 6.2 & 6.5 & 5.5 & 0.55 \\
\hline Surgical complications no. (\%) & $11(13.3)$ & $9(14.1)$ & $2(10.5)$ & 0.69 \\
\hline Pros thesis complications no. (\%) & 67 (80.7) & $49(76.6)$ & $18(94.7)$ & 0.08 \\
\hline Median HRS score & 9.73 & 9.6 & 10.2 & 0.4 \\
\hline TEP speech rehabilitation no. (\%) & $57(68.7)$ & $43(67.2)$ & $14(73.7)$ & 0.59 \\
\hline
\end{tabular}




\subsection{Tracheoesophageal Puncture and Voice Prosthesis}

Sity-four patients (77.1\%) had primary TEP and only 19 patients (22.9\%) had secondary TEP for voice restoration (Table 1). A secondary puncture was performed in mean 25.4 months after surgery (ranging from 7 to 132 months).

Postoperative complications occurred in 39 patients (47.0\%). The most common were pharyngocutaneous fistula in 18 patients (21.7\%), respiratory infection in 9 patients $(10.8 \%)$ cervical cellulitis in 6 patients (7.2\%) and cervical infection in 2 patients (2.4\%).

A total of 275 Provox ${ }^{\circledR}$ prosthesis were used for these 83 patients during this period, with a mean 3.4 devices per patient.

The mean device lifetime for the entire group was 9.8 months (ranging from 1 to 45 months), 10.4 months for patients who had undergone primary PTE and 8.0 months for patients who had undergone secondary PTE. The difference was statistically significant $(P=0.01$, MannWhitney's U-test).

The mean lifetime of the first voice prosthesis was 11.6 months (ranging from 1 to 74 months) and for the subsequent prosthetic valves was 6.2 months (from 1 to 24 months). The difference was statistically significant ( $\mathrm{P}=0.004$, nonparametric Wilcoxon test).

The Kaplan-Meier survival curve of first prosthetic valve is shown in Figure 1. The estimated mean survival of the first voice prosthesis was 11.96 months, being 12.7 months in primary PTE and 9.41 months in secondary PTE (Log Rank, $\mathrm{P}<0.05$ ). The 6 and 12 months survival of first voice prosthesis was $68.1 \%$ and $38.8 \%$ respectively.

The mean device lifetime was 6.3 months for patients who didin't undergo RT treatment and 10.6 months for patients who undergone this treatment ( $P=0.05$, MannWhitney's U-test).

In univariate analysis, no significant difference in device lifetime was associated with age $(\mathrm{P}=0.56$, nonparametric Mann-Whitney's U-test), comorbidity ( $\mathrm{P}=$ 0.08, nonparametric Mann-Whitney's U-test), primary disease $(\mathrm{P}=0.32$, nonparametric Mann-Whitney's Utest), staging ( $\mathrm{P}=0.81$, nonparametric Mann-Whitney's U-test) nor in primary resection $(\mathrm{P}=0.53$, nonparametric Mann-Whitney's U-test).

Applying the Cox proportional hazards model, there

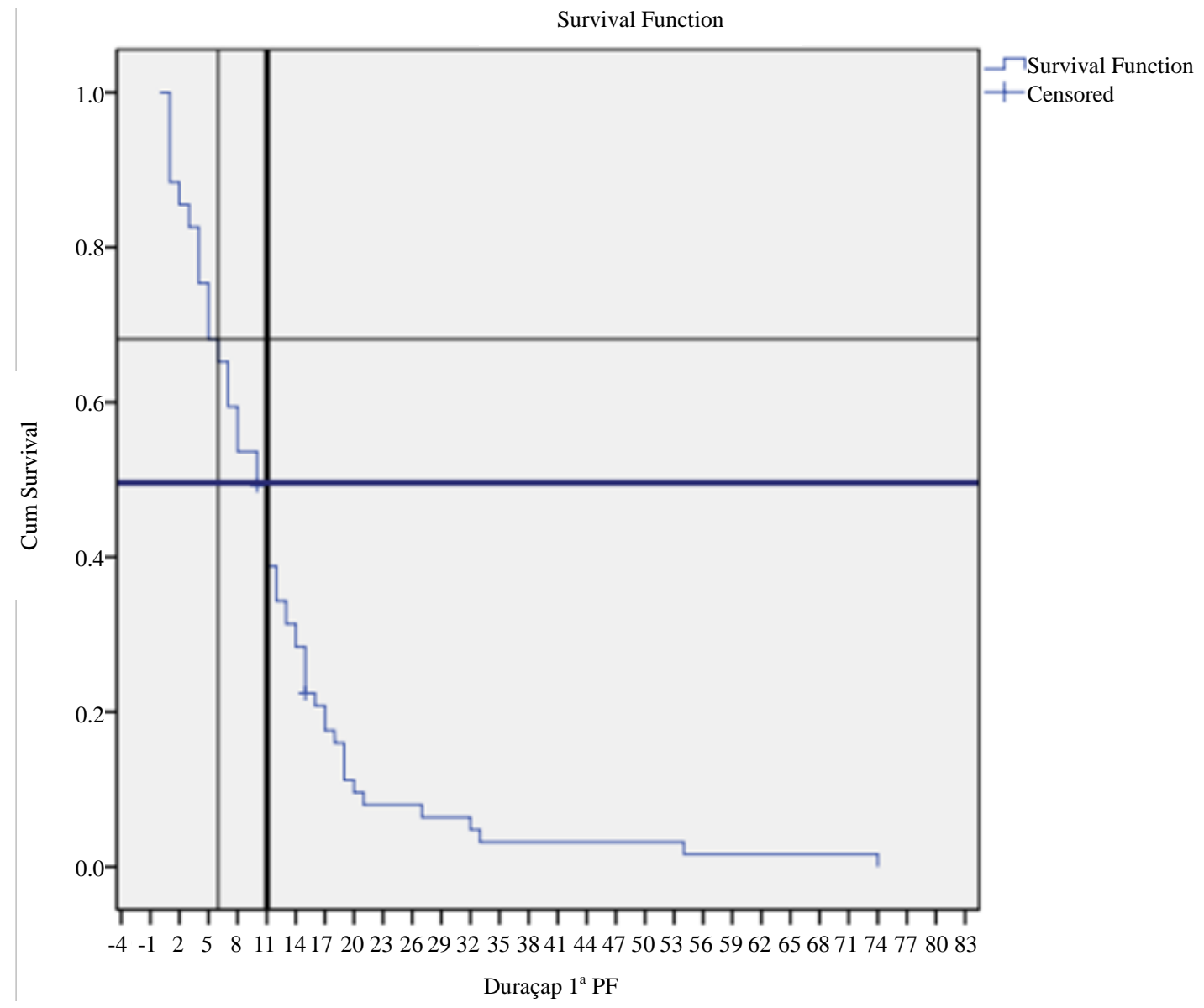

Figure 1. Kaplan-Meier proportion of survival of first prosthesis lifetime. 
RT was identified as a significant predictor of prosthetic valve failure $(\mathrm{P}=0.018$; relative hazard ratio, $1.5 ; 95 \%$ confidence interval, 1.1 - 2.4).

\subsection{Surgical and Prosthesis-Related Complications}

Surgical complications occurred in 11 of 83 patients (13.3\%) (See Table 1). When analysed by subgroup, surgical complications occurred in 9 of the 64 primary TEP patients (14.1\%) and in 2 of the 19 secondary TEP patients (10.5\%) $(\mathrm{P}=0.69$, nonparametric Mann-Whitney's U-test).

The most common surgical related complication was a large and deep tracheotomy hole in 6 patients (7.2\%) and pharyngoesophageal stricture in 5 patients $(6.0 \%)$.

In univariate analysis, no significant difference in the rate of surgical complications was associated with age ( $\mathrm{P}$ $=0.09$, nonparametric Mann-Whitney's U-test), comorbidities ( $\mathrm{P}=0.24$, nonparametric Mann-Whitney's U-test), primary disease $(\mathrm{P}=0.51$, nonparametric Mann-Whitney's U-test), staging ( $\mathrm{P}=0.17$, nonparametric MannWhitney's U-test), primary resection ( $\mathrm{P}=0.79$, nonparametric Mann-Whitney's U-test) nor with radiotherapy treatment $(\mathrm{P}=0.84$, nonparametric Mann-Whitney's U-test).

Prosthesis-related complications occurred in 67 out of 83 patients (80.7\%) (See Table 1). When analyzed by subgroup, prosthesis-related complications occurred in 49 of the 64 primary TEP patients $(76.6 \%)$ and in 18 out of the 19 secondary TEP patients (94.7\%) $(\mathrm{P}=0.08$, nonparametric Mann-Whitney's U-test). Main complications were prosthesis leakage which occurred in 63 patients $(75.9 \%)$, and prosthesis displacement which occurred in 18 patients (21.7\%). Forty-seven patients (56.6\%) developed prosthesis-related complications due to the prosthesis device and in 42 patients (50.6\%) due to PTE.

Fifty-four patients (81.8\%) who underwent radiotherapy and 13 patients (76.5\%) who hadn't undergone this treatment had prosthesis-related complications $(\mathrm{P}=0.62$, nonparametric Mann-Whitney’s U-test).

Eighteen patients (69.2\%) under 60 years old and 49 patients with an age over 60 years (86.0\%) had prosthesis complications ( $\mathrm{P}=0.07$, nonparametric Mann-Whitney's U-test).

In univariate analysis, no significant difference in rate of prosthesis-related complications was associated with comorbidity ( $\mathrm{P}=0.37$, nonparametric Mann-Whitney's U-test), primary disease ( $\mathrm{P}=0.42$, nonparametric MannWhitney's U-test), staging ( $\mathrm{P}=0.90$, nonparametric MannWhitney's U-test) nor with primary resection $(\mathrm{P}=0.93$, nonparametric Mann-Whitney's U-test).

In 4 patients $(4.8 \%)$ occurred spontaneous closure of
TEP occurred after extrusion of their prosthetic valve. One of these patients had subsequent puncture and reinsertion of the prosthesis. Two patients (2.4\%) had surgical closure of tracheoesophageal fistula due to persistent leakage. Following closure, only 1 patient opted for further TEP one year later.

\subsection{Vocal Rehabilitation}

Mean follow-up period was of 50.7 months (4.2 years) ranging from 6 to 204 months.

$68.7 \%$ of patients $(n=57 / 83)$ achieved functional tracheoesophageal speech (HRS score 10) ,67.2\% (n = 43/64) had performed primary TEP and 73.7\% ( $=$ 14/19) had performed secondary (See Table 2). The difference were not statistically significant $(\mathrm{P}=0.59$, nonparametric Mann-Whitney's U-test).

The success rate was $80.8 \%(n=21 / 26)$ in patients younger than 60 years and $63.2 \%(n=36 / 57)$ in those older than 60 years $(\mathrm{P}=0.11$, non-parametric MannWhitney's U-test).

In patients who effectuate radiotherapy treatments the success rate was $70.6 \%(n=12 / 17)$ while in patients without rathiotherapy the sucess rate was $68.2 \%$ ( $n=$ 45/66) $(P=0.85$, non-parametric Mann-Whitney's U test).

In univariate analysis no significant difference in the success rate was detected in relation to comorbidity $(\mathrm{P}=$ 0.59, nonparametric Mann-Whitney's U-test), primary disease $(\mathrm{P}=0.55$, nonparametric Mann-Whitney's Utest), staging ( $\mathrm{P}=0.08$, nonparametric Mann-Whitney's U-test) nor with primary resection $(\mathrm{P}=0.68$, nonparametric Mann-Whitney's U-test).

The mean of HRS rating scale was 9.73 (ranging from 1 to 13 points), 9.59 in primary TEP and 10.21 in secondary TEP $(\mathrm{P}=0.36$, non-parametric Mann-Whitney's Utest). A total overall score of 12 or higher was observed in 31 patients (37.3\%). Twenty-two patients (34.4\%) had performed primary TEP and 9 (47.4\%) had performed secondary TEP $(\mathrm{P}=0.30$, non-parametric Mann-Whitney's U-test).

The mean total VHI score was 37.5. Forty-two patients (50.0\%) of patients achieved excellent voice quality perceptually, 32 patients $(50.0 \%)$ had performed primary TEP and 10 patients (52.6\%) had performed secondary TEP ( $\mathrm{P}=0.59$, non-parametric Mann-Whitney's U-test).

Out of the 26 patients (31.3\%) who had unsuccessful TEP speech, a physical cause could be identified in 21 patients: a large and deep tracheostoma that led to incompetent digital occlusion $(n=9)$, chronic obstructive pulmonary disease and ineffective respiratory support (n $=5)$, pharyngoesophageal stricture $(n=5)$ and poor general conditions and impaired hand-eye coordination ( $\mathrm{n}=$ 2). In the other 5 patients, no obvious physical cause was 
Table 2. Outcome of TEP speech rehabilitation.

\begin{tabular}{|c|c|c|c|}
\hline & $\begin{array}{l}\text { No (\%) of } \\
\text { Patients }\end{array}$ & $\begin{array}{l}\text { Succesful Voice } \\
\text { Rehabilitation }\end{array}$ & $P$ value \\
\hline Age (years) & & & 0.11 \\
\hline$<60$ & 26/83 (31.3) & 21/26 (80.8) & \\
\hline$\geq 60$ & $57 / 83(68.7)$ & $36 / 57$ (63.2) & \\
\hline Comorbidity no. (\%) & & & 0.59 \\
\hline $\mathrm{ASA} \leq 3$ & 64/83 (77.1) & $43 / 64(67.2)$ & \\
\hline ASA $>3$ & 19/83 (22.9) & $14 / 19(73.7)$ & \\
\hline Tumor site no. (\%) & & & 0.55 \\
\hline Larynx & 72/83 (86.7) & $50 / 72(69.4)$ & \\
\hline Hypopharynx & 11/83 (13.3) & 6/11 (54.5) & \\
\hline Staging no. (\%) & & & 0.08 \\
\hline$\leq \mathrm{III}$ & 30/83 (36.1) & $17 / 30(56.7)$ & \\
\hline$>$ III & 53/83 (63.9) & 40/53 (75.5) & \\
\hline Resection no. (\%) & & & 0.68 \\
\hline $\mathrm{TL}$ & 63/83 (75.9) & 44/63 (69.8) & \\
\hline PLT & 20/83 (24.1) & $13 / 20(65.0)$ & \\
\hline Time of TEP no. (\%) & & & 0.59 \\
\hline PTEP & $64(77.1)$ & $43 / 64(67.2)$ & \\
\hline PTES & 19 (22.9) & $14 / 19(73.7)$ & \\
\hline Radio therapy no. (\%) & & & 0.85 \\
\hline No & 17/83 (20.5) & $12 / 17(70.6)$ & \\
\hline Yes & 66/83 (75.9) & 45/66 (68.2) & \\
\hline
\end{tabular}

identified. As alternative method of communication, one patient uses the esophageal speech and the remaining relied on writing.

A heat and moisture exchanger (HME) device was used by 52 patients (62.7\%). A Provox Free-Hands HME valve allowing "hands-free speech" was used by 1 patient (1.2\%).

Ten patients (12.0\%) died of locoregional failure or distant metastasis.

\section{Discussion}

Laryngectomy is an effective treatment for larynx carcinoma, however the subsequent inability of verbal communication has a profound impact on the quality of life and psychosocial aspect of a patient [2]. Although esophageal and electrolaryngeal speech are viable options for voice rehabilitation, TEP has been considered the most effective method, providing improved quality of life, speech intelligibility and quality, and patient satisfaction $[3,7]$.

\subsection{Vocal Rehabilitation}

Success rates for voice prosthesis rehabilitation vary from $50 \%$ to $90 \%$ in published literature. Different success rates may be explained by a heterogeneous patient population and a different or deficient evaluation system [8]. For most authors long-term success rate was defined, as the use of tracheoesophageal speech consistently or for the majority of verbal communication needs. In the present study the multiparametric voice prosthesis HRS Rating Scale was used.

In the present study, $68.7 \%$ of our patients achieved functional tracheoesophageal speech, a result that appeared very satisfactory and similar to previously reported studies [2,3,5].

We found no statistically significant tendency for an higher success rate in secondary TEP (73.2\% vs $67.2 \%)$, this contrasts with most published results which have demonstrated that the two procedures were equally successful in restoring voice (52\% to $89 \%$ vs $56 \%$ to $94 \%$ ) [4]. A possible explanation could be a selection bias since patients who underwent secondary puncture were selected more accurately (adequate evaluation of the neurological and psychological status of the patient, endoscopic and radiological explorations of the pharyngoesophageal tract, good patient motivation). Another important factor is the higher motivation of these patients after an aphonic period. However primary TEP has the advantage of avoiding a second surgical intervention, allowing early voice restoration with a considerable psychological impact.

Relatively to age, like in other published studies we verified a higher success rate of TEP speech rehabilitation in patients younger than 60 years old. A reduced vital capacity of lung and the comorbidities affecting hand-eye coordination in elderly patients probably contributed to the limited use of TEP speech in the older age group. Also, hearing loss, decreased patient motivation and unwillingness to learn have been described as possible causes of unsuccessful vocal rehabilitation. However the success rate of prosthetic valve rehabilitation can be increased by selecting younger patients with good hand-eye coordination and little comorbidity, particularly chronic obstructive pulmonary disease. Since assessment of learning capacity can be subtle, we consider that age is not a contraindication for TEP speech rehabilitation. In patients with poor dexterity, the Valved Provox Heat and Moisture Exchanger can improve the stomal occlusion. In our study, only one patient used a Provox FreeHands HME automatic tracheostome valve system, due to the lower overall economic status of our patients.

Radiotherapy has not effected vocal rehabilitation. For some authors RT is a limiting factor for a successful voice restoration, to others, RT had no consequences on 
quality of voice or complication rates [1]. However a temporary deterioration of the voice quality is expected in primary TEP due to local mucositis and tracheitis during postoperative irradiation. No manipulation of prosthesis and an electrolarynx speech is advised until resolution of acute radiation reaction.

We believe that the low socioeconomic status of some patients had an integral part in the failure of some of the cases since the patients had no means of acquiring different types of adhesive and base plates, tracheotomy tubes or buttons to fix occlusion problems of large and deep tracheostome.

\subsection{Surgical and Prosthesis-Related Complications}

TEP is not entirely perfect. Several studies reported that the rate of complication ranged from $15 \%$ to $72 \%$ [5]. In our study, we verified that prosthesis related-complications were higher (80.7\%) but easily manageable.

The existing literature data regarding the relation between timing of TEP and prosthesis related-complications are not consistent. Some authors found similar complications rate between primary and secondary TEP, while others found a higher rate of complications in patients undergoing primary TEP [4,7]. In our study patients in the secondary TEP groupdid have higher incidence of prosthesis-related complications. Most of these patients effectuated ratiotherapy treatments after TL, so TEP is placed in fibrotic field altered by ratiotherapy.

Similarly to what was verified in our study, several investigators have identified radiotherapy as a risk factor to the development of complications after TEP. Postirradiation hyposalivation with consequent modification of pharyngeal microflora could promote biofilm formation and subsequent voice device dysfunction. On the other hand, several studies have found that ratiotherapy had no effect on vocal rehabilitation or complication.

Major complications reported were leakage through or around the prosthesis (75,9\%) and prosthesis dislodgement (21,7\%), which are complications also described in several studies [5]. Leakage through the prosthesis heralds valve failure, probably due to the infection caused by Candida specimens, and usually requires replacement of the prosthesis. We had $50.6 \%$ of the patients suffered leakage through the prosthesis. Nystatin suspension on a brush can be used liberally in cleaning the device in order to limit possible colonization by candida [7]. Leakage around the prosthesis occurs because of either thinning of the tracheoesophageal wall or fistula widening by downsizing the prosthesis, placing a silicone ring or removing the device and inserting a small nasogastric tube through the fistula to allow the fistula to contract spontaneously. Two patients with intractable leakage needed surgical closure of TEP.

Future investigations will be valuable in defining and investigating strategies that may reduce complications. Patient education for early signs of complications and ways to address them show additional promise.

\subsection{Tracheosophageal Puncture and Voice Prosthesis}

Hilgers et al. described that the average device lifetime of the Provox ${ }^{\circledR}$ voice prosthesis was more than 5 months. In our study, the median device lifetime of the voice prosthesis was 9.78 months, which is longer than those reported in other series. This may be due to differences in dietary habits, body build, genetic factors, and the prevalence of gastroesophageal reflux. Therefore, less stasis of food residue around the prosthetic valves with less candida colonizing around the silicone material may lead to longer device lifetime [2]. We also found that the first prosthetic valves last longer (11.6 months) than all other subsequent prosthetic valves (6.2 months). This is probably due to TEP fragility due to voice prosthesis changes.

The Kaplan-Meier survival curve of the first prosthetic valve showed that the estimated median survival of the first voice prosthesis was 11.96 months.

Radiotherapy can influence voice prosthesis lifetime due to poor healing of the tracheoesophageal fistula in tissues compromised by this treatment [2]. This explains the shorter voice prosthesis lifetime in patients submitted to secondary TEP, mostly already submitted to RT. In our study, patients with RT treatment had longer lifetime devices probably due to the lower use of it during the treatments.

Older patients had a shorter device lifetime probably due to poor dexterity and motivation in cleaning the prosthetic valves [2].

\section{Conclusions}

Because of its safety and simplicity, tracheoesophageal puncture has become the state of the art method for voice rehabilitation after total laryngectomy.

This study adds further evidence that surgical voice restoration with prosthetic valve by TEP provides a high rate of successful voice rehabilitation in most laryngectomized patients. Despite a lower rate of successful voice restoration with primary TEP, it may be preferable for several reasons, longer lifetime voice device, lower rate of complications and no need for a second surgery. Older age can shorten the lifetime of the prosthesis, increase the rate of complications and diminish the rate of success for TEP speech rehabilitation. A patient's socioeconomic status is another factor that can limit the TEP success rate. However, if a careful screening is made selecting patients with good dexterity, motivation and capacity to learn 
results can be very satisfactory.

\section{REFERENCES}

[1] P. Boscolo-Rizzo, F. Zanetti, S. Carpené and M. C. Da Mosto, "Long-Term Results with Tracheoesophageal Voice Prosthesis: Primary versus Secondary TEP," European Archives of Oto-Rhino-Laryngology, Vol. 265, No. 1, 2008, pp. 73-77. http://dx.doi.org/10.1007/s00405-007-0423-9

[2] P. K. Lam, et al., "Long-Term Performance of Indwelling Tracheoesophageal Speaking Valves in Chinese Patients Undergoing Laryngectomy," Archives of Otolaryngology -Head and Neck Surgery, Vol. 131, No. 11, 2005, pp. 954-958. http://dx.doi.org/10.1001/archotol.131.11.954

[3] T. Terada, et al., "Voice Rehabilitation with Provox2 Voice Prosthesis Following Total Laryngectomy for Laryngeal and Hypopharyngeal Carcinoma," Auris Nasus Larynx, Vol. 34, No. 1, 2006, pp. 65-71. http://dx.doi.org/10.1016/j.anl.2006.09.017

[4] E. Cheng, et al., "Outcomes of Primary and Secondary Tracheoesophageal Puncture: A 16-Year Retrospective Analysis," Ear, Nose \& Throat Journal, Vol. 85, No. 4, 2006, pp. 262,264-267.

[5] A. Bozec, "Results of Vocal Rehabilitation Using Tracheoesophageal Voice Prosthesis after Total Laryngectomy and Their Predictive Factors," European Archives of OtoRhino-Laryngology, Vol. 267, No. 5, 2010, pp. 751-758. http://dx.doi.org/10.1007/s00405-009-1138-x

[6] M. A. Hotz, A. Baumann, I. Schaller and P. Zbären, "Success and Predictability of Provox Prosthesis Voice Rehabilitation,” Archives of Otolaryngology-Head and Neck Surgery, Vol. 128, No. 6, 2002, pp. 687-691. http://dx.doi.org/10.1001/archotol.128.6.687

[7] P. S. Dayangku Norsuhazenah, et al., "Complications Following Tracheoesophageal Puncture: A Tertiary Hospital Experience,” Academy Medicine of Singapore, Vol. 39, No. 7, 2010, pp. 565-564. 